\title{
Definition and Identification of Patients with Treatment-Resistant Depression in Real-World Clinical Practice Settings Across Asia
}

This article was published in the following Dove Press journal: Neuropsychiatric Disease and Treatment

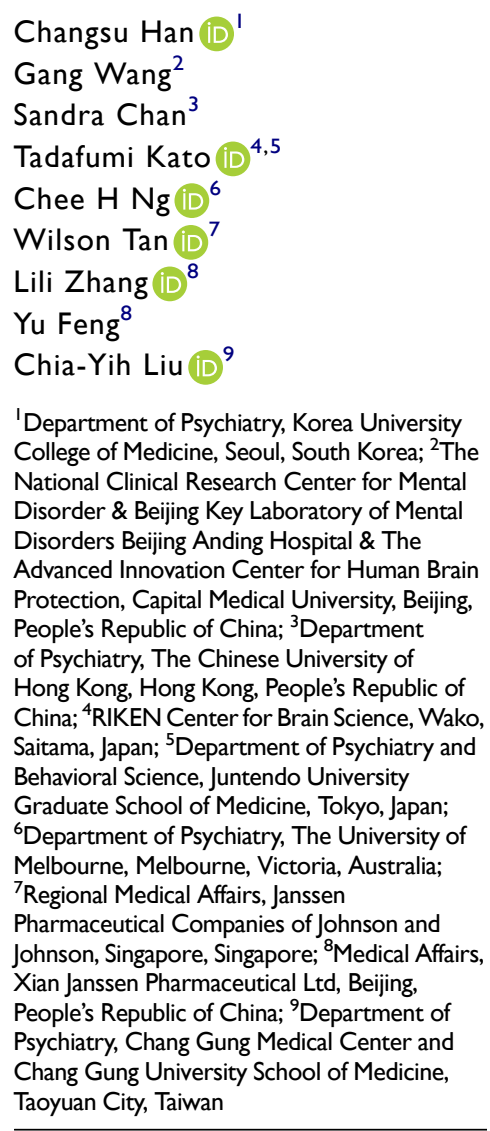

Correspondence: Chia-Yih Liu Department of Psychiatry, Chang Gung Medical Center and Chang Gung University School of Medicine, No. 5, Fuching Street,

Guishan District, Taoyuan City, Taiwan

Tel +886-3-328I200 Ext 2439

Email liucy752@cgmh.org.tw

Wilson Tan

Regional Medical Affairs, Janssen Pharmaceutical Companies of Johnson and Johnson, 2 Science Park Drive, \#07-13, Ascent, Singapore Science Park I, Singapore I I8222, Singapore

Tel +65-69187930

Email wtan27@its.jnj.com
Purpose: An Asia-Pacific expert consensus defined treatment-resistant depression (TRD) as failure of $\geq 2$ antidepressants given at adequate doses for 6-8 weeks during a major depressive episode. A survey examined how TRD was being diagnosed in real-world practices across Asia. An expert panel then interpreted the results and provided practical recommendations.

Methods: Between March and July 2018, 246 clinicians from Hong Kong, Japan, Mainland China, South Korea, and Taiwan were surveyed on how they identified TRD patients according to their own definitions.

Results: Most physicians described antidepressant failure as "no response" (79\%) or "inadequate response" (82\%); fewer chose "failure to achieve remission" (45\%). About $40 \%$ did not routinely use clinical tools to assess response. Around $52 \%$ defined adequate dose target as achieving the label's upper dose limit. About 58\% would treat for 4-8 weeks before determining antidepressant failure. Most (76\%) required the $\geq 2$ qualifying antidepressant failures to be from different classes. Approximately $60 \%$ considered antidepressant failure(s) from previous depressive episode(s) when diagnosing TRD.

Conclusion: Considering the survey results, antidepressant failure can be defined as a failure to achieve remission, or more practically as $<50 \%$ improvement in depressive symptoms or inability to return to work/study, and confirmed with a clinical tool. TRD diagnosis also requires $\geq 2$ qualifying antidepressant failures within the same depressive episode; from the same or different classes; and achieving at least the minimum effective antidepressant dose for 6-8 weeks.

Keywords: Asia, treatment-resistant depression, diagnosis, management

\section{Introduction}

Major depressive disorder (MDD) is a significant public health issue in Asia. According to the World Health Organization (WHO), almost a fifth of the world's population suffering from MDD live in Asia-Pacific countries. ${ }^{1}$ MDD is a complex and heterogeneous disease, with a pathophysiology that is not fully understood. ${ }^{2}$ In the Diagnostic and Statistical Manual of Mental Disorders (DSM-V) published by the American Psychiatric Association (APA), MDD is characterized by the following symptoms: depressed mood; markedly diminished interest or pleasure; increase or decrease in either weight or appetite; insomnia or hypersomnia; psychomotor agitation or retardation; fatigue or loss of energy; feeling of worthlessness or inappropriate guilt; diminished ability to think or concentrate, or indecisiveness; 
and recurrent thoughts of death or recurrent suicidal ideation. $^{3,4}$ To be diagnosed with MDD, at least five of the above symptoms must be present during the same twoweek period representing a change from previous functioning and one of the symptoms must be depressed mood or loss of interest or pleasure. The diverse symptom combinations that qualify for a diagnosis of MDD give rise to symptom variability among individuals diagnosed with MDD. This could have led to a wide range of pharmacologic and non-pharmacologic treatment options for MDD demonstrating varied, and sometimes limited, efficacy.,

The challenges in treating MDD are further aggravated by a subgroup of MDD patients who do not respond adequately despite multiple courses of different antidepressants. This condition is termed as treatment-resistant depression (TRD). ${ }^{7}$ However, attempts to establish a consistent definition of TRD across the globe have made little progress over the last few decades. ${ }^{8,9}$ There are also limited data on the epidemiology of TRD in the Asia-Pacific region. Recent estimates of TRD prevalence, extracted from insurance databases, among pharmaceutically treated depressive patients ranged from $4.2 \%$ in Korea to $20.9 \%$ in Taiwan. ${ }^{10,11}$ Given the lack of standardized definition of TRD, it is not surprising to observe wide-ranging differences in TRD prevalence estimates. The landmark study, Sequenced Treatment Alternatives to Relieve Depression (STAR*D), was one of the first significant studies which helped to better characterize the patients with TRD. ${ }^{12}$ The STAR*D trial observed a marked decrease in patients achieving remission with subsequent therapies after failing to respond to two initial courses of adequate dose-duration antidepressant treatments. Approximately $30 \%$ of MDD patients eventually failed to achieve remission despite multiple lines of antidepressant therapies. ${ }^{13}$ The results of STAR*D triggered discussions on various aspects of TRD definition, like the exact number of treatment failures or duration of therapy to constitute adequate treatment, that extends to current day debates. ${ }^{14,15}$

A systematic review of the published literature between 2010 and 2016 also revealed inconsistencies in the definition of TRD in both clinical and research settings in the AsiaPacific region. ${ }^{16}$ The authors of the review eventually arrived at a consensus on the definition of TRD: failure of at least two antidepressant trials, given at adequate doses, for 6-8 weeks while ensuring adequate treatment adherence, during an MDD episode. It remains unknown whether such a definition is consistent with or can be implemented in real-world clinical settings across Asia. The aim of this article is two-fold. Firstly, a survey was conducted to examine how TRD patients were being diagnosed and identified by Asian physicians in day-to-day clinical practice. Secondly, an expert panel was then convened to interpret the survey results and provide practical recommendations. The findings of this article are presented in a three-part format, comprising 1) Results of the cross-sectional survey involving physicians from multiple Asian countries, 2) Discussion of survey results among an expert panel within the context of published literature, and 3) Expert recommendations on how TRD patients could be identified in realworld clinical settings in Asia.

\section{Materials and Methods Cross-Sectional Survey Survey Sites}

A cross-sectional survey of practicing physicians recruited from South Korea (39 sites), Taiwan (30 sites), Mainland China (20 sites), Japan (17 sites) and Hong Kong (15 sites) was conducted between March and July 2018. Depending on a country's health-care system, the sites and institutions selected were actively managing patients presenting with depression. The physicians from these institutions were required to have substantial clinical experience treating depression, particularly MDD (see below "Participants").

\section{Survey Participants}

In Asia, MDD may be managed by various physician types, ranging from general practitioners to specialist psychiatrists across various country health-care systems. Therefore, regardless of the physician types, physicians who were responsible for drug treatment decisions for symptoms of depression, particularly those who treated MDD patients, were included in the survey. Included physicians must have $\geq 5$ years of clinical experience in treating MDD. In addition, participating physicians must be treating MDD patients in a typical month with $\geq 1$ patient having TRD (based on the physicians' own definition). Potential participants were invited to participate via e-mails, telephone calls, or face-to-face meetings. Those who accepted the invitation were then screened for eligibility. Informed consent was obtained from each participant prior to conducting the face-to-face survey.

\section{Survey Data Collection}

The survey consisted of open-ended, close-ended, and semi-structured questions. The interview started with 
open-ended questions like: "What does treatment-resistant depression mean to you? How would you describe it in your clinical practice?". These questions were intended to elicit spontaneous responses from the participants without being influenced by the subsequent close-ended or semistructured questions that assessed the following areas in TRD: assessment of antidepressant treatment response; criteria for defining treatment failure and TRD; dosing and treatment duration for antidepressant trials; other considerations when defining TRD like adherence, class(es) of antidepressant used and significance of previous episodes of treatment failures. Participants were not informed about the survey sponsor to avoid sponsor bias. The survey was conducted using the official language of each country. Responses were then recorded in the local language using paper and pen before being translated to English during data entry. The questionnaires were administered by trained interviewers, from a contract research organization, with at least 5 years of experience in conducting such surveys. The interviewers were trained on the overall rationale and objectives of the questionnaire, the logical sequence and intent of each question, and survey confidentiality.

\section{Survey Data Analysis}

Descriptive analyses were conducted using Stata version 15. ${ }^{17}$ Qualitative data were recorded and transcribed verbatim. Transcripts were then reviewed independently by two members of the research team while using NVivo version $11^{18}$ to identify and code salient themes. Discrepancies in coding were resolved by enlisting a third research team member to achieve consensus and were recoded.

\section{Survey Ethics Review}

This survey was approved by the institutional review boards of the Chinese University of Hong Kong (Hong Kong), the Japanese Association of the Promotion of State-of-art in Medicine (Japan), Anding Hospital (Mainland China), Korea University Ansan Hospital (South Korea), and Chang Gung Medical Center (Taiwan).

\section{Expert Panel}

A panel, comprising six psychiatry experts from the AsiaPacific region, all of whom are authors of this article, was established to discuss the survey results and make recommendations on TRD diagnosis and management in realworld clinical practice. The panel members were selected based on their extensive clinical experience and their being recognized as scientific experts in the management of TRD in their countries. A series of virtual meetings were organized to facilitate discussions among the panelists and achieve consensus on their expert recommendations.

\section{Results and Discussion Cross-Sectional Survey: Responder Characteristics}

Of the 478 physicians who were screened and deemed eligible, $246(51 \%)$ were "responders" who consented and eventually participated in the survey. Among the survey responders, a larger proportion were male (responders: male $(60 \%)$ vs females $(37 \%), \mathrm{p}<0.001)$ and worked in the private sector (responders: private sector $(66 \%)$ vs public sector $(37 \%)$, $\mathrm{p}<0.001)$. Of the 246 responders, 80 (33\%) were from Mainland China, 65 (26\%) from South Korea, 60 (24\%) from Taiwan, 24 (10\%) from Hong Kong and 17 (7\%) from Japan (Table 1). About 54\% of the responders were selfreported as "senior consultant or above", all of whom were practicing psychiatrists. The median number of years of psychiatric practice was 18 (interquartile range, IQR: 12-24) years, while the median number of years managing MDD patients was 15 (IQR: 9-21) years. In a typical month, the mean percentage of MDD consultations that were TRD patients was about $19.3 \%$.

\section{Question I: What Does TRD Mean to You? How Would You Describe It in Your Clinical Practice? Survey Results}

Majority of the responders would consider the following as key features of TRD: patients clinically diagnosed with MDD, treated with two or three antidepressants, "sufficient dose" of treatment, "sufficient period" of treatment (between 2 and 8 weeks), "no satisfactory treatment effect", or "proven ineffective". Some responders were more specific and characterized TRD patients as those who demonstrated $<50 \%$ improvement in the baseline Hamilton Depression Rating Scale (HAMD-17) or the Montgomery-Åsberg Depression Rating Scale (MADRS) scores. A few also mentioned the lack of a standard TRD definition, which tended to vary across clinical practices and research. Some further described TRD as being "hardto-cure", "complicated and refractory" and having "poor response" to "pharmacological and non-pharmacological treatment". The cause of TRD was also described as 
Table I Characteristics of Responders

\begin{tabular}{|l|l|}
\hline Demographic Characteristics & n (\%) \\
\hline $\begin{array}{l}\text { Gender } \\
\text { Male }\end{array}$ & $182(74.0)$ \\
Type of practice & $64(26.0)$ \\
Public & $129(53.8)$ \\
Private & $110(45.8)$ \\
Others & $1(0.4)$ \\
\hline Location & \\
Mainland China & $80(32.5)$ \\
South Korea & $65(26.4)$ \\
Taiwan & $60(24.4)$ \\
Hong Kong & $24(9.8)$ \\
Japan & $17(6.9)$ \\
\hline Current designation & \\
Senior Consultant or above & $132(53.7)$ \\
Consultant & $45(18.3)$ \\
Associate Consultant & $41(16.7)$ \\
Others & $28(11.4)$ \\
\hline Extent of TRD Experience & Mean (SD) \\
\hline Duration of clinical practice (years) & $19(7.7)$ \\
Duration of managing MDD (years) & $17.1(7.9)$ \\
\hline $\begin{array}{l}\text { Total number of patient consults in a typical } \\
\text { month }\end{array}$ & $412(244.3)$ \\
Percent of MDD patients, out of total patients & $31 \%(16.5 \%)$ \\
\hline
\end{tabular}

Note: ${ }^{\text {a }}$ esponder spent an equal amount of time in the public and private practice. Abbreviations: MDD, major depressive disorder; SD, standard deviation; TRD, treatment-resistant depression.

multifactorial, involving interactions between physiological, environmental and social factors.

\section{Discussion}

This open-ended qualitative question explored the spontaneous responses of Asian physicians regarding TRD definition in their day-to-day clinical practice. Various features of TRD definition were described, including the number of antidepressant treatment trials, antidepressant dosage, treatment trial duration and treatment response. The subjectivity of assessments (like "sufficient", "ineffective", "satisfactory" and "poor") is likely to aggravate further the already lack of a standard TRD definition recognized by the survey participants. ${ }^{16}$ Hence, in addition to developing a consensus on TRD definition, it is also important to standardize how TRD patients are being identified practically in the realworld clinical setting, in-line with the TRD definition.

\section{Expert Recommendations}

A recent systematic review and expert consensus defined TRD as a condition affecting MDD patients with failure to $\geq 2$ antidepressant therapies given at adequate doses for 6-8 weeks during a major depressive episode. ${ }^{16}$ In addition, attempts should be made to further clarify certain terms, like "failure" or "adequate", to ensure TRD is being described in a consistent fashion.

\section{Question 2: Antidepressant Treatment Trial - What is the Clinical Criteria for "Antidepressant Failure"? \\ Survey Results}

Participants were asked a semi-structured question about the "clinical criteria of treatment failure in MDD in clinical practice" and could select all the answers (shown in Table 2) that applied. Most physicians considered "inadequate/diminished response" (82\%) or "no response" (79\%) as indicators of antidepressant failure, with no clear distinction between them when asked about their definitions of these terms. Some participants described "inadequate/ diminished response" as a negligible improvement of symptoms and "no response" as a complete lack of symptom improvement. A few physicians specified "no response" as $<50 \%$ improvement in the HAMD-17 score.

A smaller proportion (45\%) of surveyed physicians deemed antidepressant failure as a failure to achieve remission. However, the understanding of "failure to achieve remission" was inconsistent among the surveyed physicians. Some described it subjectively as a "persistence of relevant symptoms", while others defined

Table 2 Clinical Criteria as Indicator of Antidepressant Failure

\begin{tabular}{|l|l|}
\hline Clinical Presentation & n (\%) \\
\hline $\begin{array}{l}\text { No response } \\
\text { Yes }\end{array}$ & $194(78.9)$ \\
No & $52(21.1)$ \\
\hline $\begin{array}{l}\text { Inadequate/diminished response } \\
\text { Yes }\end{array}$ & $201(81.7)$ \\
No & $45(18.3)$ \\
\hline $\begin{array}{l}\text { Failure to achieve remission } \\
\text { Yes }\end{array}$ & $110(44.7)$ \\
No & $136(55.3)$ \\
\hline $\begin{array}{l}\text { Others } \\
\text { Yes }\end{array}$ & $2(0.8)$ \\
No & $244(99.2)$ \\
\hline
\end{tabular}


it more objectively as $<80 \%$ score improvement from baseline as measured by clinical tools (eg, HAMD-17).

\section{Discussion}

According to the APA guidelines, the treatment goal of MDD should be remission of the episode and alleviation of functional impairments to improve quality of life. ${ }^{19}$ Residual symptoms of depression portend early relapse and increased recurrence rates. ${ }^{20}$ Remission involves the resolution of depressive symptoms as described by the fifth edition of the Diagnostic and Statistical Manual of Mental Disorders. ${ }^{3}$ A recent systematic review with a global expert panel has also characterized the clinical presentation of antidepressant failure, especially in clinical research, as "a lack of complete remission (ie, non-remission)" defined by a "HAMD-17 score of $>7$ or a MADRS score of $>10$ ". ${ }^{16}$ However, the same panel also suggested a more practical definition of treatment failure, which may fit better into routine clinical practice where there are time constraints: "an inability to return to work/study or failure to achieve at least 'moderate improvement' as part of the Clinical Global Impression (CGI)". ${ }^{16}$ This practical definition is less stringent than the APA guidance on remission as a treatment goal but continues to focus on functional and symptomatic improvements. It also reduces the likelihood of misclassifying a case as an antidepressant failure when the patient has responded significantly to treatment but have yet to achieve remission. This practical recommendation is consistent with our survey findings, which showed that more than half (55\%) of the Asian physicians did not use "failure to achieve remission" as an indicator of antidepressant failure. Instead, the majority (about 96\%) of survey respondents characterized antidepressant failure as "no response" or "inadequate/diminished response" or both.

Several assessment tools, including clinician-rated scales (eg, HAMD-17, MADRS, CGI), and patient-rated scales [eg, Patient Health Questionnaire-9 (PHQ-9) or Quick Inventory for Depressive Symptomatology (QIDS-16)], have clear thresholds for defining treatment "response". ${ }^{21,22}$ A few guidelines and emerging consensus defined "response" as $\geq 50 \%$ reduction in depressive symptoms, ${ }^{5,14,23,24}$ with some suggesting a lower threshold of around $25 \%$ as a clinically meaningful improvement for "highly treatment-resistant" patients. ${ }^{25}$ This is consistent with the definition, generally adopted in Asia-Pacific research settings, of "no response" as having a $<50 \%$ improvement in HAMD-17. ${ }^{16}$ Some within a recent global expert panel were also open to a criteria of $<10-20 \%$ improvement of HAMD-17 or MADRS as definition of "no response" or "treatment failure". ${ }^{16}$ The 2012 Taiwanese guidelines for MDD management ${ }^{26}$ further classified a $<25 \%$ improvement as "poor response" and a $25-$ $50 \%$ improvement as "inadequate response", in-line with the work of Fava and Davidson. ${ }^{27}$ A CGI-Improvement score of $\leq 3$ is considered an improvement. ${ }^{28}$ With the QIDS-16, a score of $\leq 5,6-8$ and $\geq 9$ correspond to remission, partial response and inadequate response, respectively. ${ }^{29}$ The PHQ9 assesses changes in score from baseline and a decrease of $\geq 5$ points, $2-4$ points and $0-1$ point versus baseline corresponds to "adequate", "probably inadequate" and "inadequate" treatment responses. ${ }^{22}$

\section{Expert Recommendations}

In keeping with the APA guidance on remission as a treatment goal, physicians should maintain a high level of suspicion for antidepressant treatment failure when an MDD patient fails to achieve remission clinically (defined according to DSM-V criteria as a failure to achieve $\geq 2$ months with no symptoms or only one or two symptoms to no more than a mild degree). Suspected antidepressant failures should be further confirmed by a clinician-rated tool such as CGI (score $>3$ ), HAM-D (score of $>7$ ) or MADRS (score of $>10$ ).

Recognizing the standard of remission may be challenging to implement in the real-world setting, it is reasonable to also define antidepressant treatment failure more practically as a lack of clinical response with $<50 \%$ (no substantial) improvement in the severity of depressive symptoms or an inability to return to work/study. A diagnosis of antidepressant failure can then be confirmed with clinician-rated tools such as CGI (score $>3$ ), $<50 \%$ reduction in HAMD-17 or MADRS.

Where there are significant time constraints, validation with patient self-rated tools like PHQ-9 changes from baseline (adequate response: $\geq 5$ points, probably inadequate: $2-4$ points and inadequate: 0 or 1 point) and QIDS-16 score (remission: $\leq 5$, partial response: $6-8$ and inadequate response: $\geq 9$ ), may also be acceptable.

\section{Question 3: Antidepressant Treatment Trial - How Should Treatment "Response" Be Assessed in Day-to-Day Clinical Practice? Is It Practical? Is It Realistic? Survey Results}

Almost all (96\%) physicians relied on clinical judgement, while only $60 \%$ of them utilized clinical tools to assess 
treatment response in MDD patients (Table 3). Among Asian physicians who used clinical tools, the most commonly used tools were the HAMD-17 (57\%), followed by the Beck Depression Inventory (BDI; 22\%).

\section{Discussion}

Clinical practice guidelines, such as those from the APA, ${ }^{19}$ the United Kingdom National Institute for Health and Care Excellence (NICE), ${ }^{30}$ the Canadian Network for Mood and Anxiety Treatments (CANMAT), ${ }^{24,31}$ recommend clinicianrated scales (eg, CGI, HAMD-17, and MADRS) as well as patient self-rated scales (eg, PHQ-9, QIDS-16, BDI and Zung Self-Rating Depression Scale [SDS]) for assessing antidepressant treatment response. Our survey indicated that some Asian physicians also used similar assessment tools. However, the use of clinical tools has not been universally adopted among Asian physicians in their day-to-day clinical practice. Around $40 \%$ of participants in this survey did not routinely use clinical tools to assess antidepressant response. In a study conducted in the United States, more than half of the psychiatrists reported that they never or rarely used scales to monitor outcomes of depression treatment. ${ }^{32}$ Similarly, $69 \%$ of the primary care physicians in Canada did not utilize standardized assessments for depression severity. ${ }^{33}$ Some key barriers to the use of clinical tools include time constraints and a lack of tools that are culturally validated and easy to administer. ${ }^{33,34}$ Several guidelines recommend using validated patient self-rated scales, such as PHQ-9 and QIDS-16. ${ }^{19,34}$ These are especially useful when there are significant time constraints.

\section{Expert Recommendations}

Ideally, antidepressant treatment response should be monitored using clinician-administered or patient self-rated

Table 3 Use of Clinical Tools to Assess Treatment Response

\begin{tabular}{|l|l|}
\hline Use of Clinical Tools & n (\%) \\
\hline No & $99(40.2)$ \\
\hline Yes & $147(59.8)$ \\
Hamilton Depression Rating Scale (HAMD- I7) & $84(57.1)$ \\
Beck Depression Inventory (BDI) & $33(22.5)$ \\
Zung Self-Rating Depression Scale (SDS) & $19(12.9)$ \\
Diagnostic and Statistical Manual of Mental Disorders & II (7.5) \\
(DSM) & \\
Patient Health Questionnaire-9 (PHQ-9) & $9(6.1)$ \\
Clinical Global Impressions Scale (CGIs) & $8(5.4)$ \\
Montgomery-Åsberg Depression Rating Scale & $6(4.1)$ \\
(MADRS) & \\
\hline
\end{tabular}

scales to optimize treatment outcomes. However, in busy clinical practices across Asia, exercising clinical judgement to assess antidepressant response in routine practice is acceptable. Antidepressant failure determined based on clinical judgement should be further confirmed using a clinician-administered scale (eg, CGI, HAMD-17 or MADRS). Otherwise, validated patient self-rated scales (eg, PHQ-9 or QIDS-16) may also be considered when there are significant time constraints.

\section{Question 4: Antidepressant Treatment Trial - What is the Target Dose? Survey Results}

Almost all (96\%) physicians considered achieving optimal dose as being an integral part of an adequate antidepressant treatment trial (Table 4). Should a patient remain symptomatic, about half (52\%) of the surveyed physicians would continue to up-titrate the antidepressant dose until its upper limit is reached per prescribing information (ie, "optimal dose"). About $24 \%$ of physicians equate "achieving the dose range (per prescribing information)" to "optimal dose".

\section{Discussion}

A majority (76\%) of respondents prescribed the antidepressant dose according to label recommendations. Around 52\% of the surveyed physicians may actively up-titrate the antidepressant dose to its upper limit, per label recommendations, to achieve

Table 4 Achieving and Defining Optimal Dose in Antidepressant Treatment Trial

\begin{tabular}{|l|l|}
\hline Dosing in Antidepressant Treatment Trial & $\mathbf{n}(\%)$ \\
\hline $\begin{array}{l}\text { Achieve optimal dose as part of adequate } \\
\text { treatment }\end{array}$ & \\
Yes & $236(95.9)$ \\
No & $3(1.2)$ \\
This is not a criterion & $7(2.9)$ \\
\hline $\begin{array}{l}\text { Definition of optimal dose } \\
\text { Achieve the upper limit of dose as per label }\end{array}$ & $127(51.6)$ \\
recommendation & \\
Achieve the dose range as per label recommendation & $58(23.6)$ \\
Maximum tolerated dose & $44(17.9)$ \\
Achieve the lower limit of dose as per label & $2(0.8)$ \\
recommendation & \\
Others a & $5(2.0)$ \\
This is not a criterion & $7(2.8)$ \\
\hline
\end{tabular}

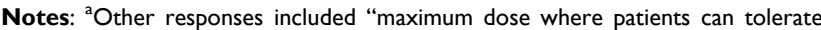
adverse events" $(n=I)$, "sufficient dosage to improve patient's condition and restoring function" $(n=I)$, "maximum tolerated dose not exceeding the recommended limit" $(n=1)$, "titrate upwards according to response until maximum tolerated dose" $(n=I)$, and "effective tolerated dose within the recommended limit" $(n=1)$. 
better efficacy while balancing patient safety and tolerability. A recent systematic review on the definition of TRD documented multiple studies indicating at least a "minimum dose of $150 \mathrm{mg}$ /day of imipramine equivalents" as the "optimal dose" for antidepressant treatment trial. ${ }^{16}$ The initial adult dosage of imipramine (brand name: Tofranil-PM) ranges from 100 to $300 \mathrm{mg}$ per day in hospitalized patients, and 75 to $200 \mathrm{mg}$ per day in the outpatient setting, for the relief of symptoms of depression. ${ }^{35}$ By "minimum", these studies appear to suggest an opportunity for upward titration of the antidepressant to achieve "optimal dose". A few other studies included in this systematic review considered "maximum dose" as the "optimal dose" for an adequate antidepressant treatment trial. ${ }^{16}$ However, results from a multi-center pragmatic trial involving 2011 untreated MDD patients across 48 clinics in Japan may challenge this "maximum dose" paradigm, as the study found no advantage in up-titrating sertraline to $100 \mathrm{mg}$ compared with remaining on $50 \mathrm{mg} .{ }^{36}$ Several other selective serotonin reuptake inhibitor (SSRI) studies also did not show an increase in receptor occupancy or efficacy benefit with dose escalation. ${ }^{37}$ In addition to efficacy, it is also important for physicians to be vigilant about side effects when choosing and dosing antidepressants for improved tolerability, thereby enhancing treatment compliance.

\section{Expert Recommendations}

Clinical practice guidelines and published evidence suggest a "minimum dose of $150 \mathrm{mg}$ /day imipramine equivalents" as the "optimal dose" for antidepressant treatment trial. Should a patient remain symptomatic, it is reasonable for physicians to consider up-titrating the antidepressant dose, as permitted by its prescribing information, to achieve better efficacy while balancing patient safety and tolerability. Patients responding inadequately despite the "optimal dose" are deemed to have failed their antidepressant treatment trial. ${ }^{16}$

\section{Question 5: Antidepressant Treatment Trial - What is the Target Duration of Therapy? Survey Results}

As shown in Table 5, a duration of between 4 and $<8$ weeks reflected the practice of most surveyed physicians $(58 \%)$, while only $22 \%$ would keep patients on an antidepressant for 8 weeks or more.

\section{Discussion}

The target treatment duration indicated by more than half of the surveyed physicians is consistent with clinical practice guidelines (eg, APA and CANMAT), which suggest a duration of 4-8 weeks for an adequate antidepressant trial. $^{8,19,24,31}$ However, $18 \%$ of surveyed physicians would make a determination of antidepressant failure in $<4$ weeks. Of the 89 Asia-Pacific studies captured in a systematic literature review, 61 studies (69\%) specified the duration of the antidepressant trial. Of these 61 studies, 21 (34\%) reported $\geq 6$ weeks, $9(15 \%)$ reported $\geq 8$ weeks, and 7 (11\%) reported $\geq 4$ weeks. $^{16}$ In addition to these studies, a global expert panel also convened to review published international guidelines and eventually arrived at a consensus of 6-8 weeks as the target duration for an adequate antidepressant treatment trial. $^{16}$

\section{Expert Recommendations}

We recommend a time frame of 6-8 weeks as an adequate duration for an antidepressant treatment trial before making a determination of antidepressant failure.

\section{Question 6: Antidepressant Treatment Trial - Should Adherence Be Reasonably Assessed in Real-World Practice Before Determining Antidepressant Failure? Survey Results}

Approximately $87 \%$ of the physicians considered treatment adherence as critical for a valid antidepressant treatment trial (Table 6). About 14\% of surveyed physicians did not consider treatment adherence as important for defining treatment failure.

\section{Discussion}

Non-adherence to antidepressants, which could be determined by medication possession ratio, self-reports or length of therapy, has been shown to worsen symptom severity and

Table 5 Duration of an Antidepressant Treatment Trial

\begin{tabular}{|l|l|}
\hline $\begin{array}{l}\text { Treatment Duration Before Defining Condition as } \\
\text { Treatment Failure }\end{array}$ & $\mathbf{n ~ ( \% )}$ \\
\hline$<4$ weeks & $44(17.9)$ \\
4 to $<6$ weeks & $81(34.3)$ \\
6 to $<8$ weeks & $56(23.7)$ \\
8 to $<10$ weeks & $18(7.6)$ \\
10 to $<12$ weeks & $21(8.9)$ \\
$\geq 12$ weeks & $12(5.1)$ \\
Others & \\
\hline
\end{tabular}

Notes: ${ }^{a}$ Other responses included " 2 to 12 weeks depending on patient's condition" $(n=1)$, "switch to another medication if patient has side effects within 2 to 4 weeks" $(n=I)$, "2 to 4 weeks for hospitalized patients and 4 to 6 weeks for outpatients" $(n=1)$, and "half year" $(n=1)$. 
reduce treatment response and remission rates. ${ }^{38}$ As per the APA and the CANMAT guidelines, adherence to therapy is one of the cornerstones of optimal measurement-based care. ${ }^{19,24,31}$ The APA guidelines recommend the two-item self-administered Patient Adherence Questionnaire (PAQ) to assess the degree of adherence and establish the reasons for deviating from the recommended dose (if any). ${ }^{19}$

\section{Expert Recommendations}

Ensuring treatment adherence is critical for an antidepressant treatment trial to be valid. Physicians should avoid characterizing a patient as having an antidepressant failure or making a serious diagnosis of TRD without first confirming that the patient is adherent to the prescribed antidepressant therapy.

\section{Question 7: TRD Diagnosis - How Many Antidepressant Failures Must a MDD Patient Experience Before Diagnosing TRD?}

\section{Survey Results}

More than $95 \%$ of the physicians defined TRD as $\geq 2$ treatment failures, with more than half $(57 \%)$ diagnosing TRD once a patient experiences two treatment failures. Around $39 \%$ of the responders considered TRD only after three treatment failures (Table 7).

\section{Discussion}

Almost all physicians agreed that TRD involves $\geq 2$ antidepressant failures, with more than half of them setting the minimum at two treatment failures for a diagnosis of TRD. These survey findings are consistent with a recently conducted systematic review and expert consensus. ${ }^{16}$ While the majority (71\%) of the studies in the systematic review defined TRD as $\geq 2$ antidepressant failures, the second biggest group (16\%) reported TRD as failure to $\geq 1$ antidepressant. About $9 \%$ of the studies defined TRD as $\geq 3$ antidepressant failures. Several clinical practice guidelines, such as those from the World Federation of Societies of Biological Psychiatry (WFSBP), ${ }^{39}$

Table 6 Consideration of Adherence in Antidepressant Treatment Trial

\begin{tabular}{|l|l|}
\hline $\begin{array}{l}\text { Ensuring Adherence Before Defining Treatment } \\
\text { Failure }\end{array}$ & $\mathbf{n}(\%)$ \\
\hline Yes & $201(87.4)$ \\
No & $14(6.1)$ \\
This is not a criterion & $19(8.3)$ \\
\hline
\end{tabular}

the British Association for Psychopharmacology (BAP) ${ }^{40}$ and the Royal Australia and New Zealand College of Psychiatrists $(\text { RANZCP) })^{41}$ also defined TRD as a failure to respond to $\geq 2$ adequate antidepressant treatment trials.

The STAR*D trial found that the likelihood of MDD patients achieving remission significantly decreased after two antidepressant treatment failures and the condition would require a more complex medication regimen. ${ }^{42}$ TRD, if defined more stringently (eg, $\geq 3$ antidepressant failures), may result in a delay in diagnosing and initiating treatment(s) for this difficult-to-treat population, thereby leading to poorer outcomes.

\section{Expert Recommendations}

Physicians should consider a diagnosis of TRD if their MDD patients experience $\geq 2$ antidepressant treatment failures.

\section{Question 8: TRD Diagnosis - Should the Target Duration of Therapy Be the Same for Each Trial Before Diagnosing TRD? Survey Results}

More than two-thirds (69\%) of the physicians responded that the duration of each antidepressant treatment trial should be similar, while $16 \%$ of physicians would treat their patients with a new antidepressant for a longer duration following each treatment failure (Table 8).

\section{Discussion}

The time frame for antidepressant treatment trial has been discussed above. This question aimed to better understand whether Asian physicians, before diagnosing TRD, would adjust the duration of subsequent antidepressant treatment trials, should their patient demonstrate antidepressant failure in the current episode. A study on treatment patterns of TRD using commercial claims in the United States reported a shorter duration for each line of therapy (LOT) in TRD episodes compared to corresponding LOT in non-TRD episodes. However, there was no observable trend towards progressively shortening or lengthening the treatment

Table 7 Number of Treatment Failures Before Diagnosing TRD

\begin{tabular}{|l|l|}
\hline The Minimum Number of Treatment Failures & $\mathbf{n}(\%)$ \\
\hline I treatment failure & $5(2.0)$ \\
2 treatment failures & $139(56.5)$ \\
3 treatment failures & $86(35.0)$ \\
4 treatment failures & $4(1.6)$ \\
$>4$ treatment failures & $6(2.4)$ \\
This is not a criterion & $6(2.4)$ \\
\hline
\end{tabular}


duration for each subsequent LOT within non-TRD episodes as well as TRD episodes. ${ }^{43}$ The majority (69\%) of the surveyed physicians in this study maintained a similar duration for antidepressant treatment trials regardless of LOT. Besides, the STAR*D trial showed that moderately aggressive dosing for $\geq 8$ weeks was necessary with firstand second-line antidepressant treatment in order to achieve remission in MDD patients. ${ }^{42}$ There is currently no study comparing the benefits of keeping the same treatment duration across LOT versus actively shortening or lengthening the treatment duration with each subsequent LOT.

\section{Expert Recommendations}

MDD patients should be afforded $\geq 2$ adequate antidepressant treatment trials before diagnosing them as having TRD. ${ }^{16}$ Before a TRD diagnosis, the target duration of antidepressant treatment trials in MDD patients should remain the same for between 6 and 8 weeks. For patients already diagnosed with TRD, the duration for antidepressant treatment trials will be discussed in a separate article that focuses on TRD management.

\section{Question 9: TRD Diagnosis - Must the Two Qualifying Antidepressant Failures Occur Within the Same Current MDD Episode? Does Antidepressant Failure(s) in Previous MDD Episode(s) Count? Survey Results}

Most physicians (60\%) took into account the previous episode(s) of antidepressant failure(s) when defining TRD. Around $40 \%$ would not consider previous episode(s) of antidepressant failure(s), as shown in Table 9.

Table 8 Duration of Each Line of Treatment Trial

\begin{tabular}{|l|l|}
\hline Duration & $\mathbf{n}(\%)$ \\
\hline Same & $170(69.1)$ \\
\hline $\begin{array}{l}\text { Different } \\
\text { Shorter duration in subsequent trials following each } \\
\text { failure } \\
\text { Longer duration in subsequent trials following each } \\
\text { failure } \\
\text { Others a }\end{array}$ & $21(8.5)$ \\
\hline
\end{tabular}

Notes: "Others included "depends on individual's response to the treatment" $(n=I I)$, "depends on the recommended treatment period of the medication" $(n=3)$, "treatment period is irrelevant as long as it fits a duration of 6 to 8 weeks" $(n=I)$ and "not necessary to consider the treatment duration" $(n=I)$.

\section{Discussion}

Whether the $\geq 2$ qualifying antidepressant failures must occur within a single depressive episode to diagnose TRD is not a straightforward question. One related consideration is whether TRD has been previously diagnosed or is the current depressive episode, possibly the first time TRD is being considered. Another consideration is whether TRD is a chronic condition characterized homogenously by treatment-resistant episode(s), or whether non-resistant (ie, easier-to-treat) depressive episodes can also occur in a patient previously diagnosed with TRD. If indeed all depressive episodes in TRD patients are homogenously treatment-resistant by nature, it is not unreasonable to diagnose TRD by taking into account antidepressant failure(s) from previous depressive episode(s). However, if nonresistant depressive episode(s) can also occur in patients previously diagnosed with TRD (ie, depressive episodes within the same patient are non-homogenous), then each depressive episode should be assessed independently for treatment resistance by having the $\geq 2$ qualifying antidepressant failures occur within the same episode. Ultimately, the key clinical question is whether the term TRD should be ascribed to a patient (ie, a TRD patient with homogenous treatment-resistant episodes) or to a depressive episode. Unfortunately, there is currently insufficient evidence on the TRD neurobiology, psychopathology and epidemiology to fully inform on the above discussion. Hence, it is not surprising to witness differing recommendations on the above question. A recent global expert panel specified the $\geq 2$ qualifying antidepressant failures to occur within a major depressive episode for a diagnosis of TRD. ${ }^{12}$ On the other hand, the Agency for Healthcare Research and Quality in the United States recommends including antidepressant failures from the previous episode(s) when diagnosing TRD. ${ }^{44}$

\section{Expert Recommendations}

In-line with the need to exercise extreme prudence when assigning patients with a serious diagnosis like TRD, we suggest for the $\geq 2$ qualifying antidepressant failure(s) to occur within the same depressive episode, especially for

Table 9 Consideration of Treatment Failure in Previous Episodes of MDD

\begin{tabular}{|l|l|}
\hline Treatment Failure in Previous Episodes & n (\%) \\
\hline Yes & $148(60.2)$ \\
No & $67(27.2)$ \\
This is not a criterion & $31(12.6)$ \\
\hline
\end{tabular}


patients who are being diagnosed with TRD for the first time.

\section{Question 10: TRD Diagnosis - Must the Two Qualifying Antidepressant Failures Be from Different Classes? Survey Results}

Most physicians (about 76\%) responded that the antidepressants used had to be from different classes to diagnose TRD, while about a quarter did not think that antidepressant class was important for TRD definition (Table 10).

\section{Discussion}

In the systematic review, only 37 (41\%) of the 87 studies explored the class(es) of antidepressants when defining TRD. ${ }^{16}$ Almost all $(n=35)$ of these studies required the qualifying antidepressant failures to be from different classes. Nonetheless, there is currently limited evidence and still no widely acknowledged consensus on whether different classes of antidepressant treatment trials are required for diagnosing TRD. ${ }^{15}$ Due to the unique properties, including pharmacokinetics and pharmacodynamics of individual drugs, a patient may respond differently to different antidepressants, even when they belong to the same class. Moreover, the responsiveness to a specific antidepressant or class of antidepressants also depends on the individual's genetic makeup. This is supported by a growing body of evidence demonstrating the clinical benefits of pharmacogenomic-guided medication selection. ${ }^{45,46}$ Hence, physicians should not presume all patients with antidepressant failures to naturally fail other antidepressants from the same class. The APA guidelines recommend switching to another antidepressant of the same or a different class, when a patient responds inadequately to her/his current antidepressant. ${ }^{19}$ This is also consistent with the guidance from Stahl's Essential Psychopharmacology: The Prescriber's Guide (4th ed.). ${ }^{47}$

\section{Expert Recommendations}

TRD patients, who are likely to fail multiple lines of treatment, should be afforded as many antidepressant

Table 10 Need for Antidepressant Treatment Failures from Different Classes

\begin{tabular}{|l|l|}
\hline Antidepressants from Different Classes & $\mathbf{n}(\%)$ \\
\hline Yes & $186(75.6)$ \\
No & $33(13.4)$ \\
This is not a criterion & $27(11.0)$ \\
\hline
\end{tabular}

options as possible. For the diagnosis of TRD, antidepressants used for treatment trials can be from the same or different classes.

\section{Strengths and Limitations}

Surveyed physicians from the selected five major Asian countries/regions may not be representative of physicians from the entire Asia region, with its diverse health systems and practices. The subjectivity of open-ended questions and their responses are inherent limitations to such surveys. Moreover, how physicians respond verbally to a survey may not always correspond with their behaviors in real-world clinical practice.

To our knowledge, this is the first survey that recruited physicians across several Asian countries from various practice types (eg, public vs private) to understand the management of TRD across Asia. The survey was conducted in each country's official language to enhance the uniformity of understanding the questionnaires and their responses among participants. Even among experts, there is little consensus on how TRD should be defined and how such patients could be identified in real-world clinical settings. ${ }^{8,9}$ Hence, despite the above limitations, this survey yielded important information on how TRD was being managed by Asian physicians in their day-to-day clinical practice. In fact, this survey revealed, to a varying extent, discrepancies in different aspects of TRD management between the expert recommendations and how Asian physicians are practicing in the real-world. Our results may help guide future physician educational activities.

\section{Conclusion}

Majority of physicians used "no/inadequate response" as an indicator of antidepressant failure compared to $45 \%$ who adopted the standard of "failure to achieve remission". Measurement of treatment response with clinical tools was also not a routine for majority of Asian physicians. About half of the physicians defined adequate dose as achieving the upper limit of the label dose recommendation, while a quarter of them aimed to fall within the dose range. Fifty-eight percent of Asian physicians would treat their patients for 4-8 weeks before determining antidepressant failure; but around $20 \%$ of them may treat for $<4$ weeks. Most physicians required the $\geq 2$ qualifying antidepressant failures to be from different classes. Sixty percent of physicians considered antidepressant failure(s) from previous depressive episode(s) when diagnosing TRD. 
In-line with the APA guidance on remission as a treatment goal, antidepressant treatment failure should be defined clinically as failure to achieve remission. Taking into account the survey results, a more practical definition of $<50 \%$ improvement in depressive symptoms or inability to return to work/study is also acceptable. A clinician-rated tool should be used to confirm treatment failure before making a definitive and serious diagnosis of TRD. Diagnosis of TRD also requires $\geq 2$ qualifying antidepressant trials, from the same or different classes, achieving at least the minimum dose of $150 \mathrm{mg} /$ day imipramine equivalents for 6-8 weeks, and occur within the same depressive episode.

\section{Acknowledgment}

The article comprised of two parts: Firstly, a crosssectional survey was conducted to describe how TRD was being diagnosed and managed by Asian physicians in the real-world setting. Secondly, an expert panel was convened to interpret the survey results and provide practical recommendations. We confirm informed consent was obtained from participants in both the survey and the expert panel. We further confirm the cross-sectional survey complied with the Declaration of Helsinki. We thank IQVIA Asia-Pacific for collecting the data and conducting the statistical analysis.

\section{Author Contributions}

Janssen Pharmaceuticals Asia-Pacific conceived and conceptualized the study design, while data collection was conducted by IQVIA Asia-Pacific. All authors made substantial contributions to analysis and interpretation of data; took part in drafting the article or revising it critically for important intellectual content; agreed on the journal to which the article will be submitted; gave final approval of the version to be published; and agree to be accountable for all aspects of the work.

\section{Funding}

Janssen Pharmaceuticals Asia-Pacific provided funding for the data collection.

\section{Disclosure}

Chee $\mathrm{H} \mathrm{Ng}$ had served as an advisory committee member for Lundbeck, Grunbiotics, Servier, Pfizer and Eli Lilly, received research grant support from Pfizer and Lundbeck, and speaker honoraria from Janssen Asia-Pacific, Servier, Lundbeck, Bristol-Myers Squibb, Organon, Eli Lilly,
GlaxoSmithKline, Astra-Zeneca and Pfizer. Chia-Yih Liu served on advisory boards for Eli Lilly, Janssen, Pfizer, GlaxoSmithKline, and Servier; and received grant/ research support from Eli Lilly, Janssen, Otsuka, Sumitomo and Sanofi-Aventis; is a member of the speakers' bureaus for AstraZeneca, Eli Lilly, GlaxoSmithKline, Janssen, Otsuka, Pfizer, Sanofi-Aventis, and Servier. Changsu Han is a member of speakers' bureaus for Lundbeck, Otsuka, Lilly Korea, Pfizer Korea, Janssen Korea; and received research support from the Department of Public Health \& Welfare of South Korea, Otsuka Korea, Eisai, and AB Biotics. Gang Wang reports receiving grants outside of the submitted work from Chiatai Tianqing, Dainippon Sumitomo Pharma, Eli Lilly, GlaxoSmithKline, Jiangsu Hansoh, Otsuka Pharmaceutical, Shandong Luye, ShiJiaZhuang No 4 Pharm, and Xi'an Janssen. Tadafumi Kato reports receiving personal fees outside of the submitted work from Agilent Technologies, Astellas, Boehringer Ingelheim, Dainippon Sumitomo Pharma Co., Ltd., Eli Lilly, GlaxoSmithKline, Janssen, Kyowa Hakko Kirin Co., Ltd, Kyowa Pharmaceutical Industry Co., Ltd, Meiji Seika Pharma Co., Ltd., Mochida Pharmaceutical Co., Ltd., MSD, Otsuka Pharmaceutical Co., Ltd., Pfizer, Shionogi \& Co., Ltd., Taisho Toyama Pharmaceutical Co., Ltd., Wako Pure Chemical Industries, and Yoshitomiyakuhin. He also reports grants and personal fees from Takeda outside of the submitted work. Lili Zhang, Wilson Tan, and Yu Feng are employees of Janssen Asia-Pacific. Wilson Tan holds shares in Janssen Asia-Pacific awarded as part of his total compensation package. The authors report no other conflicts of interest associated with this work.

\section{References}

1. Depression and other common mental disorders: global health estimates; 2017. Available from:https://www.who.int/mental_health/ management/depression/prevalence_global_health_estimates/en/. Accessed November 18, 2019.

2. Pratt R, Stapelberg NJC. Early warning biomarkers in major depressive disorder: a strategic approach to a testing question. Biomarkers. 2018;23(6):563-572. doi:10.1080/1354750X.2018.1463563

3. American Psychiatric Association. Diagnostic and Statistical Manual of Mental Disorders. 5th ed. 2013.

4. De Berardis D, Fornaro M, Orsolini L, et al. Alexithymia and suicide risk in psychiatric disorders: a mini-review. Front Psychiatry. 2017;8:148. doi:10.3389/fpsyt.2017.00148

5. Cipriani A, Furukawa TA, Salanti G, et al. Comparative efficacy and acceptability of 21 antidepressant drugs for the acute treatment of adults with major depressive disorder: a systematic review and network meta-analysis. Lancet. 2018;391(10128):1357-1366. doi:10.1016/S0140-6736(17)32802-7 
6. Dirmaier J, Steinmann M, Krattenmacher T, et al. Nonpharmacological treatment of depressive disorders: a review of evidence-based treatment options. Rev Recent Clin Trials. 2012;7 (2):141-149. doi: $10.2174 / 157488712800100233$

7. Berlim MT, Turecki G. What is the meaning of treatment resistant/ refractory major depression (TRD)? A systematic review of current randomized trials. Eur Neuropsychopharmacol. 2007;17 (11):696-707. doi:10.1016/j.euroneuro.2007.03.009

8. McIntyre RS, Filteau M-J, Martin L, et al. Treatment-resistant depression: definitions, review of the evidence, and algorithmic approach. $J$ Affect Disord. 2014;156:1-7. doi:10.1016/j. jad.2013.10.043

9. Nierenberg AA, Amsterdam JD. Treatment-resistant depression: definition and treatment approaches. J Clin Psychiatry. 1990;51 (Suppl):39-47.

10. Kim N, Cho SJ, Kim H, et al. Epidemiology of pharmaceutically treated depression and treatment resistant depression in South Korea. PLoS One. 2019;14(8):e0221552. doi:10.1371/journal.pone.0221552

11. Fife D, Feng Y, Wang MY-H, et al. Epidemiology of pharmaceutically treated depression and treatment resistant depression in Taiwan. Psychiatry Res. 2017;252:277-283. doi:10.1016/j.psychres.2017.03.006

12. Howland RH. Sequenced treatment alternatives to relieve depression (STAR*D). Part 2: study outcomes. J Psychosoc Nurs Ment Health Serv. 2008;46(10):21-24. doi:10.3928/02793695-20081001-05

13. Warden D, Rush AJ, Trivedi MH, Fava M, Wisniewski SR. The STAR*D project results: a comprehensive review of findings. Curr Psychiatry Rep. 2007;9(6):449-459. doi:10.1007/s11920-007-0061-3

14. Trevino K, McClintock SM, McDonald Fischer N, Vora A, Husain MM. Defining treatment-resistant depression: a comprehensive review of the literature. Ann Clin Psychiatry. 2014;26(3):222-232.

15. Gaynes BN, Asher G, Gartlehner G, et al. Definition of treatment-resistant depression in the medicare population. Agency for Healthcare Research and Quality (US); 2018. Available from: http://www.ncbi.nlm.nih.gov/books/NBK526366/. Accessed October 26, 2020.

16. $\mathrm{Ng} \mathrm{CH}$, Kato $\mathrm{T}$, Han $\mathrm{C}$, et al. Definition of treatment-resistant depression - Asia Pacific perspectives. J Affect Disord. 2019;245:626-636. doi:10.1016/j.jad.2018.11.038

17. Stata Statistical Software: release 15. [computer program]. College Station, TX: StataCorp LLC2017.

18. NVivo Qualitative Data Analysis Software. [computer program] version 11. QSR International Pty Ltd; 2017.

19. American Psychiatric Association. Practice guideline for the treatment of patients with major depressive disorder; 2010. Available from: https://psychiatryonline.org/pb/assets/raw/sitewide/practice_ guidelines/guidelines/mdd.pdf. Accessed November 18, 2019.

20. Judd LL, Akiskal HS, Maser JD, et al. A prospective 12-year study of subsyndromal and syndromal depressive symptoms in unipolar major depressive disorders. Arch Gen Psychiatry. 1998;55(8):694-700. doi:10.1001/archpsyc.55.8.694

21. Maust D, Cristancho M, Gray L, Rushing S, Tjoa C, Thase M. Psychiatric rating scales. In: Handbook of Clinical Neurology. Vol. 106. Elsevier; 2012:227-237.

22. Moore M, Ali S, Stuart B, et al. Depression management in primary care: an observational study of management changes related to PHQ-9 score for depression monitoring. Br J Gen Pract. 2012;62 (599):e451-457. doi:10.3399/bjgp12X649151

23. Fava M. Diagnosis and definition of treatment-resistant depression. Biol Psychiatry. 2003;53(8):649-659. doi:10.1016/s0006-3223(03) 00231-2

24. Lam RW, McIntosh D, Wang J, et al. Canadian network for mood and anxiety treatments (CANMAT) 2016 clinical guidelines for the management of adults with major depressive disorder: section 1. disease burden and principles of care. Can J Psychiatry. 2016;61(9):510-523. doi: $10.1177 / 0706743716659416$
25. Rush AJ, Kraemer HC, Sackeim HA, et al. Report by the ACNP task force on response and remission in major depressive disorder. Neuropsychopharmacology. 2006;31(9):1841-1853. doi:10.1038/sj. npp. 1301131

26. CY YTL L, Chen CS. Treatment Guideline for Major Depressive Disorder in Primary Care. Taiwan Association Against Depression; 2012.

27. Fava M, Davidson KG. Definition and epidemiology of treatment-resistant depression. Psychiatr Clin North Am. 1996;19 (2):179-200. doi:10.1016/s0193-953x(05)70283-5

28. Busner J, Targum SD. The clinical global impressions scale: applying a research tool in clinical practice. Psychiatry (Edgmont). 2007;4 (7):28-37.

29. Taylor CB. How to Practice Evidence-Based Psychiatry: Basic Principles and Case Studies. American Psychiatric Pub; 2009.

30. National Institute for Health and Care Excellence (NICE). Depression - recognition and management; 2018. Available from: https://www.nice.org.uk/guidance/cg90. Accessed November 18, 2019.

31. Kennedy SH, Lam RW, McIntyre RS, et al. Canadian network for mood and anxiety treatments (CANMAT) 2016 clinical guidelines for the management of adults with major depressive disorder: section 3 . pharmacological treatments. Can J Psychiatry. 2016;61(9):540-560. doi: $10.1177 / 0706743716659417$

32. Zimmerman M, McGlinchey JB. Why don't psychiatrists use scales to measure outcome when treating depressed patients? J Clin Psychiatry. 2008;69(12):1916-1919. doi:10.4088/jcp.v69n1209

33. Rizvi SJ, Grima E, Tan M, et al. Treatment-resistant depression in primary care across Canada. Can J Psychiatry. 2014;59(7):349-357. doi:10.1177/070674371405900702

34. Rush AJ, Bernstein IH, Trivedi MH, et al. An evaluation of the quick inventory of depressive symptomatology and the hamilton rating scale for depression: a sequenced treatment alternatives to relieve depression trial report. Biol Psychiatry. 2006;59(6):493-501. doi:10.1016/j.biopsych.2005.08.022

35. Mallinckrodt Inc. Tofranil-PM ${ }^{\mathrm{TM}}$ (imipramine pamoate) [package insert]; 2017. Available from: https://www.accessdata.fda.gov/drugsatfda_docs/ labe1/2014/017090s078lbl.pdf. Accessed November 18, 2019.

36. Kato T, Furukawa TA, Mantani A, et al. Optimising first- and second-line treatment strategies for untreated major depressive disorder - the SUND study: a pragmatic, multi-centre, assessor-blinded randomised controlled trial. BMC Med. 2018;16(1):103. doi:10.1186/ s12916-018-1096-5

37. Ruhé HG, Booij J. Evidence why paroxetine dose escalation is not effective in major depressive disorder: a randomized controlled trial with assessment of serotonin transporter occupancy. Neuropsychopharmacology. 2009;34(4):999-1010. doi:10.1038/ npp. 2008.148

38. Ho SC, Chong HY, Chaiyakunapruk N, Tangiisuran B, Jacob SA. Clinical and economic impact of non-adherence to antidepressants in major depressive disorder: a systematic review. J Affect Disord. 2016;193:1-10. doi:10.1016/j.jad.2015.12.029

39. Bauer M, Severus E, Kohler S, Whybrow PC, Angst J, Moller HJ. World federation of societies of biological psychiatry (WFSBP) guidelines for biological treatment of unipolar depressive disorders. Part 2: maintenance treatment of major depressive disorder - update 2015. World J Biol Psychiatry. 2015;16(2):76-95. doi:10.3109/ 15622975.2014.1001786

40. Cleare A, Pariante CM, Young AH, et al. Evidence-based guidelines for treating depressive disorders with antidepressants: a revision of the 2008 British association for psychopharmacology guidelines. J Psychopharmacol. 2015;29(5):459-525. doi:10.1177/ 0269881115581093

41. Malhi GS, Bassett D, Boyce P, et al. Royal Australian and New Zealand college of psychiatrists clinical practice guidelines for mood disorders. Aust N Z J Psychiatry. 2015;49(12):1087-1206. doi:10.1177/0004867415617657 
42. Gaynes BN, Warden D, Trivedi MH, Wisniewski SR, Fava M, Rush AJ. What did STAR*D teach us? Results from a large-scale, practical, clinical trial for patients with depression. Psychiatr Serv. 2009;60(11):1439-1445. doi:10.1176/ps.2009.60.11.1439

43. Kubitz N, Mehra M, Potluri RC, Garg N, Cossrow N. Characterization of treatment resistant depression episodes in a cohort of patients from a US commercial claims database. PLoS One. 2013;8(10):e76882. doi:10.1371/journal.pone.0076882

44. Agency for Healthcare Research and Quality. Definition of treatment-resistant depression in the medicare population; 2017. Available from: https://www.ahrq.gov/sites/default/files/wysiwyg/ research/findings/ta/drafts-for-review/trd-draft.pdf. Accessed November 18, 2020.
45. Greden JF, Parikh SV, Rothschild AJ, et al. Impact of pharmacogenomics on clinical outcomes in major depressive disorder in the GUIDED trial: a large, patient- and rater-blinded, randomized, controlled study. J Psychiatr Res. 2019;111:59-67. doi:10.1016/j. jpsychires.2019.01.003

46. Tanner J-A, Davies PE, Voudouris NC, et al. Combinatorial pharmacogenomics and improved patient outcomes in depression: treatment by primary care physicians or psychiatrists. $J$ Psychiatr Res. 2018;104:157-162. doi:10.1016/j.jpsychires.2018.07.012

47. Stahl S, Grady M. Stahl's Essential Psychopharmacology: The Prescriber's Guide. 4th ed. Cambridge University Press; 2011.

\section{Publish your work in this journal}

Neuropsychiatric Disease and Treatment is an international, peerreviewed journal of clinical therapeutics and pharmacology focusing on concise rapid reporting of clinical or pre-clinical studies on a range of neuropsychiatric and neurological disorders. This journal is indexed on PubMed Central, the 'PsycINFO' database and CAS, and is the official journal of The International Neuropsychiatric Association (INA). The manuscript management system is completely online and includes a very quick and fair peer-review system, which is all easy to use. Visit http://www.dovepress.com/testimonials.php to read real quotes from published authors. 